\title{
Evaluating drug resistance in visceral leishmaniasis: the challenges
}

\author{
S HENDRICKX ${ }^{1}$, PJ GUERIN ${ }^{2,3}$, G CALJON $^{1}, \mathrm{SL} \mathrm{CROFT}^{4}$ and $\mathrm{L} \mathrm{MAES}^{1}{ }^{*}$ \\ ${ }^{1}$ Laboratory for Microbiology, Parasitology and Hygiene (LMPH), University of Antwerp, Antwerp, Belgium \\ ${ }^{2}$ Nuffield Department of Clinical Medicine, Centre for Tropical Medicine and Global Health, University of Oxford, \\ Oxford, UK \\ ${ }^{3}$ Infectious Diseases Data Observatory, University of Oxford, Oxford, UK \\ ${ }^{4}$ London School of Hygiene $\Xi^{\circ}$ Tropical Medicine, Faculty of Infectious and Tropical Diseases, London, UK
}

(Received 3 August 2016; revised 21 September 2016; accepted 13 October 2016; first published online 21 November 2016)

\section{SUMMAR Y}

For decades antimonials were the drugs of choice for the treatment of visceral leishmaniasis (VL), but the recent emergence of resistance has made them redundant as first-line therapy in the endemic VL region in the Indian subcontinent. The application of other drugs has been limited due to adverse effects, perceived high cost, need for parenteral administration and increasing rate of treatment failures. Liposomal amphotericin $\mathrm{B}(\mathrm{AmB})$ and miltefosine (MIL) have been positioned as the effective first-line treatments; however, the number of monotherapy MIL-failures has increased after a decade of use. Since no validated molecular resistance markers are yet available, monitoring and surveillance of changes in drug sensitivity and resistance still depends on standard phenotypic in vitro promastigote or amastigote susceptibility assays. Clinical isolates displaying defined MIL- or AmB-resistance are still fairly scarce and fundamental and applied research on resistance mechanisms and dynamics remains largely dependent on laboratory-generated drug resistant strains. This review addresses the various challenges associated with drug susceptibility and -resistance monitoring in VL, with particular emphasis on the choice of strains, susceptibility model selection and standardization of procedures with specific readout parameters and well-defined threshold criteria. The latter are essential to support surveillance systems and safeguard the limited number of currently available antileishmanial drugs.

Key words: Visceral leishmaniasis, drug susceptibility, assay procedures, harmonization.

INTRODUCTION: THE GROWING NEED TO DEFINE RESISTANCE

Based on mortality and morbidity, leishmaniasis is currently still one of the world's most neglected tropical infectious diseases (Houweling et al. 2016) with the visceral form (VL - visceral leishmaniasis) causing approximately $0 \cdot 2-0 \cdot 4$ million cases and up to 30000 deaths worldwide annually (Alvar et al. 2012). However, the actual number of VL infected individuals is probably higher due to underreporting and delayed diagnosis (Gurunath et al. 2014). The Indian subcontinent alone was responsible for over $60 \%$ of the global VL disease burden with almost $50 \%$ of all VL cases in Bihar state (India), making it the VL 'hotspot' (Bhunia et al. 2013; Muniaraj, 2014). Although VL mainly strikes the populations in poverty in developing countries, over the years it has become an emerging problem due to a rise in migration patterns, a lack of control measures and the growing number of HIV/VL coinfections for instance (Ready, 2014). Currently, treatment of

* Corresponding author: Laboratory for Microbiology, Parasitology and Hygiene (LMPH), University of Antwerp, Faculty of Pharmaceutical, Biomedical and Veterinary Sciences, Universiteitsplein 1 - building $\mathrm{S}$ 7·27, B-2610 Antwerp, Wilrijk, Belgium. E-mail: louis. maes@uantwerpen.be human VL relies on a limited number of drugs all with issues that limit their widespread use. While VL was mainly treated with pentavalent antimony $\left(\mathrm{Sb}^{\mathrm{V}}\right.$ ) formulations in the past (Chakravarty \& Sundar, 2010; Haldar et al. 2011), their first-line use in the Indian subcontinent was largely abandoned due to the emergence of widespread $\mathrm{Sb}^{\mathrm{V}}$-resistance (Muniaraj, 2014). Although liposomal amphotericin $\mathrm{B}(\mathrm{AmB})$ (AmBisome ${ }^{\circledR}$ ) is currently recommended in the Indian subcontinent within a large-scale VL elimination effort, until recently its use was restricted due its high cost, limited availability and the requirement for cold chain facilities. At present, a large effort is being made to make AmBisome ${ }^{\circledR}$ available at largescale in endemic areas. In 2011, Gilead partnered with the World Health Organization (WHO) resulting in a large batch of AmBisome ${ }^{\circledR}$ that is now available at discounted prices in developing countries. Following the 2012 London Declaration (Balasegaram et al. 2012), it has even been donated by Gilead via the WHO, currently making it the drug of choice for the VL elimination programme in the Indian subcontinent. In addition to its enhanced availability, the KalaCORE programme (www.kalacore.org) aims to improve access and supports cold-chains in India.

In the recent past, miltefosine (MIL) monotherapy was proposed as an effective and more affordable

Parasitology (2018), 145, 453-463. C Cambridge University Press 2016. This is an Open Access article, distributed under the terms of the Creative Commons Attribution licence (http://creativecommons.org/licenses/by/4.0/), which permits unrestricted re-use, distribution, and reproduction in any medium, provided the original work is properly cited. 
alternative, while the use of paromomycin (PMM) is limited to combinations with $\mathrm{Sb}^{\mathrm{V}}$ in East Africa (Davidson et al. 2009; Thakur et al. 2000) or in combination with MIL as a proposed second-line treatment in the Indian subcontinent (Sundar et al, 2011). However, several in vitro and in vivo laboratory studies indicated that development of resistance against both drugs could be expected, even when used in combination therapy (Seifert et al. 2003; Garcia-Hernandez et al. 2012; Hendrickx et al. 2012, 2014). MIL-monotherapy was implemented until 2014 as the first-line option in the kala-azar elimination programme (Jha et al. 2013). High relapse rates combined with the possible limited drug exposure in some patients (Rijal et al. 2013; Dorlo et al. 2014) ultimately led to a revision of this current VL treatment regimen. Although no definite link with intrinsic drug resistance could be established (Rijal et al. 2013; Carnielli et al. 2014; Hendrickx et al. 2015a), the use of MIL-monotherapy is now discouraged.

In addition to challenges with drug resistance in human VL, the situation is generally comparable for canine leishmaniasis (canL). While the use of the drugs that are also used to treat human VL is discouraged for canL treatment, there has been widespread use of MIL in veterinary practice. This deserves particular attention as it creates a significant additional selection drug pressure for Leishmania infantum (Noli \& Saridomichelakis, 2014). Since no cure can be obtained, repeated treatments of infected dogs could select for resistant parasites and enhanced zoonotic transmission to man.

It is essential that in-depth research into the characterization of the factors affecting treatment efficacy and in particular identifying drug resistance mechanisms should acquire more momentum to provide molecular markers for ongoing resistance surveillance which, in turn, will give guidance to physicians and the health community to draw up adapted treatment policies. There is an urgent need to characterize and distinguish between parasite- and host-related effects on drug efficacy. Since a lot of variation in drug susceptibility has already been described between strains from different geographical regions (Hailu et al. 2010; Machado et al. 2010; ChrusciakTalhari et al. 2011; Soto et al. 2004, 2008) a reliable and reproducible method is needed to discriminate shifts in drug susceptibility and actual drug resistance within parasite populations from the host pharmacokinetic and immunological factors. Even though an intensive search for easy-to-use molecular markers has been pursued during the last decade, we still do not have standard criteria and methodologies to unequivocally define a parasite's drug susceptibility. The pivotal role of the patient (immunity, genetic background, etc.) in post-treatment relapses should not be overlooked either.
DRUG RESISTANCE MARKERS

When talking about drug resistance, it is important to distinguish between drug susceptibility, drug sensitivity and drug resistance. Drug susceptibility is defined as the response of a certain Leishmania strain/isolate to a standard drug under defined in vitro conditions, whereas drug sensitivity implies measuring the response of the strain/isolate to a standard drug in vivo using predefined doses, doseschedules, and including pharmacokinetics and immune status of the host. Ineffectiveness of killing organisms by what is considered a state-ofthe-art treatment generally refers to drug resistance. Since drug susceptibility testing is quite labourintensive, expensive and time-consuming, access to validated resistance markers that are easier to use in routine laboratory settings would be highly desirable (Croft et al. 2006; t'Kindt et al. 2010). In this respect, numerous proteomic and metabolomic studies on large sample sets of laboratory and clinical isolates currently aim to identify putative resistance markers and novel drug targets (Scheltema et al. 2010; Downing et al. 2011; Vanaerschot et al. 2012b; Berg et al. 2013; De Jesus et al. 2014). However, the results of such studies are difficult to interpret given their dependence on the parasite stage and culture conditions, and the general pleiotropic resistance mechanisms expected (Silva et al. 2011). Molecular characterization is further hampered by the highly plastic nature of the Leishmania genome, as reflected by its ability to swiftly modulate gene expression by gene amplification/deletion and to alter its chromosome ploidy in reaction to stress (Ubeda et al. 2008; Leprohon et al. 2009; Brotherton et al. 2013). Given the availability of $\mathrm{Sb}^{\mathrm{V}}$-resistant clinical isolates, numerous studies have focused on identifying molecular markers responsible for changes in Sb-susceptibility, revealing as the most reported targets aquaglyceroporin 1 (AQP1) (Mandal et al. 2010; Kumar et al. 2012) and the ATP-binding cassette (ABC) transporter MRPA (Singh, 2006; Ashutosh et al. 2007; Mittal et al. 2007; Mukherjee et al. 2007; Kumar et al. 2012), which play a role in drug uptake and sequestration. Other targets suggested to be involved in Sb-resistance are phosphoglycerate kinase (PGK) implicated in glycolysis metabolism (Kazemi-Rad et al. 2013), the multidrug-resistance protein 1 (Mukherjee et al. 2013), the kinetoplastid membrane protein (El et al. 2009), heat-shock protein 83 (HSP83) (Vergnes et al. 2007), histone H2A (Singh et al. 2010), $\gamma$-glutamylcysteine synthase (Ashutosh et al. 2012), ornithine decarboxylase (Mukherjee et al. 2007), mitogen-activated protein kinase (Ashutosh et al. 2012; Kazemi-Rad et al. 2013) and protein tyrosine phosphatase (Kazemi$\mathrm{Rad}$ et al. 2013). Since variations in gene expression do occur between different strains and species, recent studies recommend expression analysis of 


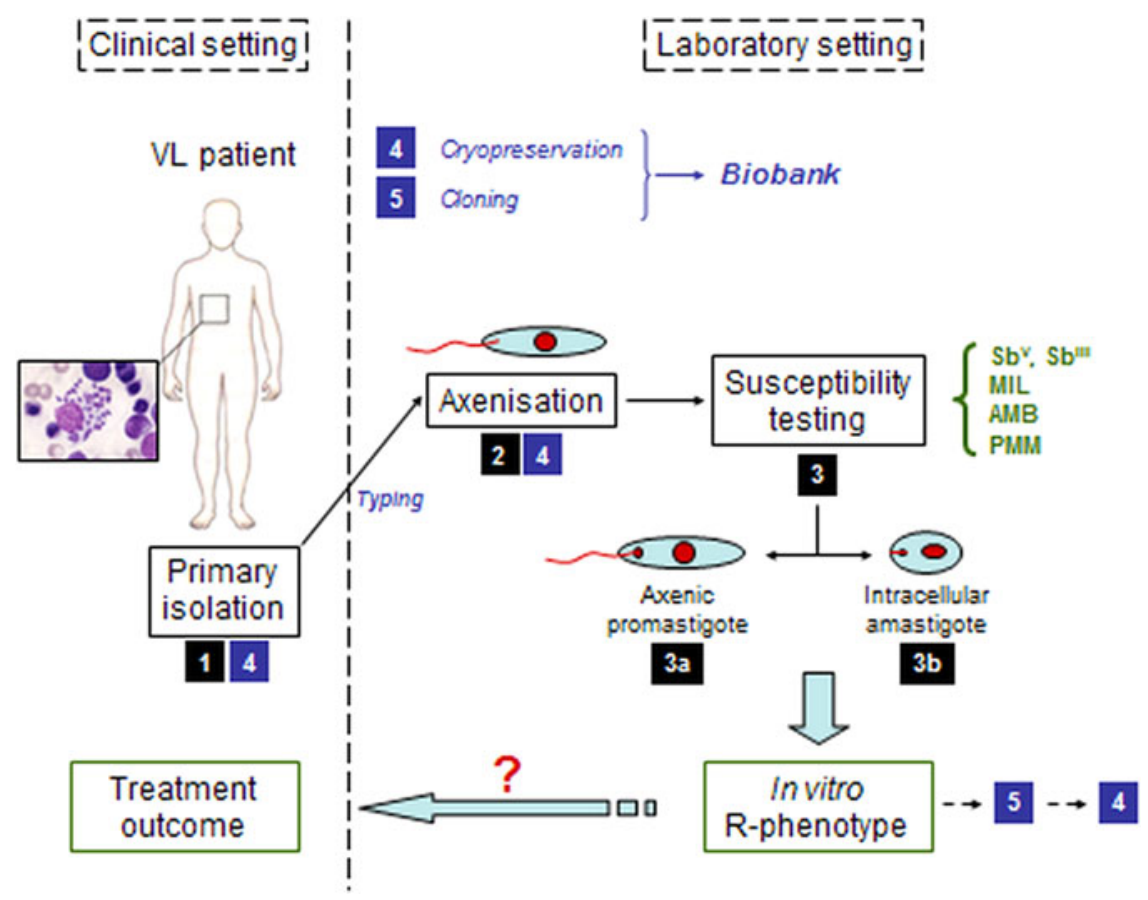

Fig. 1. Schematic overview of the pathway from the clinical setting with infected patient to the in vitro susceptibility testing of the clinical field isolate in the laboratory setting. (1) primary isolation from infected patient; (2) adaptation of the parasite to in vitro culture; (3) susceptibility testing either on a/ promastigotes or b/ intracellular amastigotes; (4) cryopreservation; (5) cloning.

multiple genes as a valid biomarker to differentiate Sb-resistance in clinical isolates (Kumar et al. 2012; Imamura et al. 2016).

For MIL, several studies suggested genetic adaptations in the Leishmania donovani putative miltefosine transporter (LdMT) and its cofactor LdRos3 (Perez-Victoria et al. 2003a, b; Perez-Victoria et al. 2006a; Seifert et al. 2007). However, identification of fully reliable molecular markers is still partly hampered by the lack of in vivo defined drug-resistant clinical isolates (Rijal et al. 2013; Mondelaers et al. 2016). Although reports on clinical resistance have appeared for the other two used drugs PMM and AmB (Purkait et al. 2012; Hendrickx et al. 2014), generally the number of clinical failure isolates associated to drug resistance is still scarce, hence most of our current knowledge on drug resistance has been gathered using laboratory-derived resistant and susceptible parasites. In addition, most laboratory studies have focused on resistanceselected promastigotes rather than the relevant intracellular amastigote stage, which may conceal the real predictive value of the findings.

\section{DRUG SUSCEPTIBILITY TESTING}

\section{Diagnosis}

Parasitological diagnosis in the patient still relies on the microscopic examination of bone-marrow or spleen aspirates accompanied by the establishment of in vitro aspirate cultures of promastigotes (Sinha et al. 1993; Mondal et al. 2010; Boelaert et al. 2014). Detection of drug resistance still relies on parasite isolation and phenotypic susceptibility testing (Sundar et al. 2014). Since these processes are complicated (Fig. 1) and are hampered by safety risks, complexity and slow generation of results (Maes et al. 2013; Boelaert et al. 2014), parasite isolation for diagnosis has been gradually replaced by non-invasive molecular techniques or immunochromatographic methods detecting Leishmania antigens or antibodies in patient sera (WHO, 2010; Boelaert et al. 2014).

\section{Microbiological testing}

Given Leishmania's digenetic life cycle, the parasite's drug susceptibility can be determined either on the promastigote vector stage or the amastigote mammalian stage. Most laboratories still routinely establish drug susceptibility of clinical isolates on the axenically cultured promastigote stage. By exposing promastigotes to serial drug dilution series, parasite viability can be read out quite easily with Alamar blue, MTT or resazurin (Fumarola et al. 2004; Maes et al. 2013). Although a correlation can be found between promastigote and amastigote susceptibility for some drugs (Kulshrestha et al. 2013), the intracellular amastigote model is still considered to be the gold standard in antileishmanial drug susceptibility determination given the stagedependent drug efficacy (Vermeersch et al. 2009). To determine the intracellular amastigote susceptibility, 
Table 1. Factors involved in the proposed standardization

\begin{tabular}{ll}
\hline \hline 1. & Species, strain of Leishmania \\
2. & Methodology \\
& A. Parasite stage \\
& B. Host cell type \\
3. & Medium \\
4. & Inoculum \\
5. & Incubation temperature \\
6. & Incubation time \\
7. & Compound/drug formulation \\
8. & Endpoint \\
9. & Quality control \\
& A. Number of replicates/repeats \\
& B. Reference drugs and acceptable range \\
\hline \hline
\end{tabular}

macrophages are infected with either metacyclic promastigotes (routinely available upon parasitological diagnosis) or organ-derived amastigotes (generally after adaptation to laboratory rodents). After infection, serial drug dilutions are added to the infected cells and the amastigote burden reduction compared to untreated control cells is determined microscopically, making this assay time-consuming, labour-intensive and therefore quite expensive. Given the stagespecific difference in drug susceptibility, the use of axenic amastigotes is still under debate as there are differences in drug susceptibility compared with the intracellular amastigote (Gupta et al. 2001; Vermeersch et al. 2009). Although assays using luminescent, fluorescent or colorimetric assays are useful for drug screening, the genetic manipulation of the isolate and time-in-culture makes this approach redundant in this context. When it comes to designing an assay for intracellular amastigotes, the use of macrophages of diverse origins (e.g. different cell lines vs primary macrophages) (Seifert et al. 2010), different culture media and the use of different methods of infection and treatment protocols all contribute to the fact that direct comparison of susceptibility data between different laboratories is virtually impossible without agreed standardization of protocols and analysis (Table 1).

\section{Defining drug resistance}

In the absence of easy ways to assess in vivo resistance due to the large number of factors affecting host response, in vitro techniques are generally considered for defining drug resistance. It specifically refers to a particular decrease of susceptibility of a certain Leishmania strain or species to a standard drug under the same predefined in vitro conditions and falsely assumes that the initial susceptibility of the parasite population before treatment is always known (Croft et al. 2006). In order to define 'in vitro' resistance, a drug concentration 'threshold' should be selected that is able to distinguish a susceptible population from a non-susceptible one. Concentration thresholds or putative molecular markers can only be defined after extensive susceptibility studies on hundreds or thousands of clinical isolates over varying regions, as has been established for malaria by the WorldWide Antimalarial Resistance Network (WWARN) (www.wwarn.org) and for drug resistant bacteria in Europe by the European Committee on Antimicrobial Susceptibility Testing (EUCAST). Tentatively proposed 'threshold' values of the current antileishmanial reference drugs $\mathrm{Sb}^{\mathrm{V}}$, AmB, MIL and PMM for all species of the L. donovani complex are summarized in Table 2 (Maes et al.2013). As an alternative, the use of an activity or resistance index has been suggested, expressing the fold decrease of susceptibility of a certain isolate compared with a drug susceptible reference isolate (Yardley et al. 2006; Inocencio da Luz et al. 2009). For some drugs, this approach is severely hampered by the natural variations in susceptibility of drugresponsive clinical isolates, precluding definition of clear cut-off values for resistance.

\section{Hurdles encountered during in vitro susceptibility testing}

Defining a resistance 'threshold' concentration is further complicated by the known variation in species and strain drug-responsiveness, making the establishment of a single 'threshold value extremely challenging. Next to these interspecies differences and the genetic diversity linked to geographical background, the polyclonal nature of strains (Fernandez et al. 2012; Hendrickx et al. 2012) also precludes extension of conclusions on susceptibility/resistance between species and hence enforces similar susceptibility studies for every Leishmania species and strain (Gouzelou et al. 2013).

Drug susceptibility testing of field isolates is logically assumed to be the most useful method to predict treatment outcome, as has been established for antibiotics in bacterial infections (Boothe, 2010) and for malaria (Duru et al. 2015). However, treatment failure is a complex interplay between various factors (Table 3) related to either drug, parasite or host, and is thus not necessarily exclusively linked to drug susceptibility (Vanaerschot et al. 2014). For example for $\mathrm{Sb}^{\mathrm{V}}$, a correlation was found between treatment outcome and the in vitro amastigote susceptibility profile of field isolates ranging from susceptible $(\mathrm{S} / \mathrm{S})$ over intermediate $(\mathrm{R} / \mathrm{S})$ to resistant $(\mathrm{R} / \mathrm{R})$. Actually, $\mathrm{R} / \mathrm{R}$ cases could be linked to non-responders or relapse cases while $\mathrm{S} / \mathrm{S}$ strains were linked to cure. The intermediate $\mathrm{R} / \mathrm{S}$ profile was linked to an increased risk for $\mathrm{R} / \mathrm{R}$ development (Inocencio da Luz et al. 2009). On the other hand for MIL, in vitro susceptibility data could not be used to predict treatment failure (Hendrickx et al. 2015a) even though 
Table 2. 'Breakpoint' estimates ${ }^{\text {a }}$ for categorizing drug-susceptibility and drug resistance against antimonials (Sb), miltefosine (MIL), paromomycin (PMM) and amphotericin B (AmB)

\begin{tabular}{|c|c|c|c|c|c|}
\hline Drug $^{b}$ & $\begin{array}{l}\text { Promastigotes } \\
\text { (axenic) } \\
\mathrm{IC}_{50}\end{array}$ & $\begin{array}{l}\text { Amastigotes (primary mouse } \\
\text { macrophages) } \\
\mathrm{IC}_{50}\end{array}$ & $\begin{array}{l}\text { Susceptibility limits }\left(\mathrm{IC}_{50}\right. \\
\text { estimates) } \\
\text { sensitive }\end{array}$ & $\begin{array}{l}\text { Cytotoxicity } \\
\text { (MRC-5) } \\
\text { resistant }\end{array}$ & $\mathrm{CC}_{50}$ \\
\hline $\mathrm{Sb}^{\mathrm{V}}$ & $>77$ & $10-15$ & $<20$ & $>70$ & $>64$ \\
\hline $\mathrm{Sb}^{\mathrm{III}}$ & $40-50$ & $5-6$ & $<15$ & $>70$ & $>64$ \\
\hline MIL & $2-5$ & $3-6$ & $<10$ & $>25^{\mathrm{c}}$ & 32 \\
\hline PMM & $15-25$ & $40-50$ & $<60$ & $>150^{\mathrm{c}}$ & $>500$ \\
\hline $\mathrm{AmB}$ & $0 \cdot 1-0 \cdot 3$ & $0 \cdot 01-0 \cdot 03$ & $<0 \cdot 5$ & $(>2)^{c}$ & $>8$ \\
\hline
\end{tabular}

a Based on results obtained with sensitive reference strains (L. donovani MHOM/ET/67/L82 and L. infantum MHOM/ MA/67/ITMAP263).

${ }^{\mathrm{b}} \mu \mathrm{g} \mathrm{mL} \mathrm{m}^{-1}$ for $\mathrm{Sb}, \mu \mathrm{M}$ for other compounds.

$c$ at present, no resistant isolates from treated patients are available yet.

Table 3. Overview of factors involved in VL disease progression and treatment failure

\begin{tabular}{|c|c|}
\hline Factor & References \\
\hline \multicolumn{2}{|l|}{ Host } \\
\hline Immunological factors & (Jarvis \& Lockwood, 2013; Ostyn et al. 2014) \\
\hline Pharmacokinetics & (Ostyn et al. 2014) \\
\hline Genetics & (Blackwell, 1996; Castellucci et al. 2014) \\
\hline Reinfection & (Rijal et al. 2013) \\
\hline Environment & (Perry et al. 2013) \\
\hline \multicolumn{2}{|l|}{ Drug } \\
\hline Drug quality & (Dorlo et al. 2012a, b) \\
\hline Intrinsic drug properties (e.g. $\mathrm{T}_{1 / 2}$ ) & (Hastings et al. 2002) \\
\hline Treatment duration & (Geli et al. 2012) \\
\hline Non-compliance & (Rijal et al. 2013) \\
\hline \multicolumn{2}{|l|}{ Parasite } \\
\hline $\begin{array}{l}\text { Lower intrinsic susceptibility to the drug } \\
\text { - gene amplification of drug target enzymes }\end{array}$ & (Singh et al. 2006; Rijal et al. 2013) \\
\hline $\begin{array}{l}\text { - decrease in intracellular drug concentration due to extrusion by } \\
\text { specific transporters }\end{array}$ & (Mondelaers et al. 2016) \\
\hline Higher parasite fitness & $\begin{array}{l}\text { (Vanaerschot et al. 2014; Hendrickx et al. } 2015 \text {; } \\
\text { Hendrickx et al. 2016) }\end{array}$ \\
\hline
\end{tabular}

some studies do suggest a weak link between in vitro susceptibility and treatment outcome (Bhandari et al. 2012; Rijal et al. 2013). Also the promastigote backtransformation assay, initially proposed as an alternative to assess treatment outcome (Hendrickx et al. 2014) could not differentiate between responder and relapse patients when evaluated on a larger set of clinical isolates (Hendrickx et al. 2015a).

Probably even more important is the fact that, although several labs worldwide have been involved in susceptibility determination of clinical isolates, drug susceptibility values remain fairly inconclusive due the lack of validated standard operating procedures (SOPs), precluding direct comparison of results between laboratories mainly due to large variation in susceptibility assay protocols and endpoints. Even for susceptibility research within the same laboratory, either for antimicrobials, antiparasitics or antifungals, results may vary significantly in time and between replicate tests (Rex et al. 1997).
It is therefore essential to improve harmonization of laboratory assays while also recognizing that this may not be straightforward. A panel of SOPs and well-defined procedures for quality control would allow better comparison of results, strengthen statistical analysis and could eventually contribute to establish well-defined endpoints and drug resistance breakpoints. For malaria, for example, a special resistance network was founded (WWARN: WorldWide Antimalarial resistance Network; www.wwarn.org; now operating under the auspices of the newly established Infectious Diseases Data Observatory IDDO) which focuses on the surveillance of drug efficacy by providing detailed procedures for drug preparation, experimental protocols, a tool to generate in vitro $\mathrm{IC}_{50}$ and $\mathrm{IC}_{90}$ values, and literature reviews thereby ensuring that all information generated on antimalarial drug resistance remains of the highest quality and are searchable in a single place (Lourens et al. 2010; Sibley \& Price, 2012; 
Table 4. Overview of the operating procedures presented in the supplementary material section

\begin{tabular}{ll}
\hline \hline Procedure & Title \\
\hline 1 & Biphasic culture media for primary isolation of promastigotes from spleen- or bone-marrow aspirates \\
2 & Monophasic culture media for axenisation of promastigotes \\
3 & Cryopreservation of Leishmania promastigotes \\
4 & Cloning of Leishmania promastigotes \\
5 & Preparation of stock solutions of antileishmania reference drugs \\
6 & Drug-susceptibility assay for promastigotes \\
7 & Drug-susceptibility assay for intracellular amastigotes \\
\hline \hline
\end{tabular}

Woodrow et al. 2013). Furthermore, the development of external quality assurance programmes has provided critical tools to compare results across laboratories (Lourens et al. 2010; Lourens et al. 2014). Unfortunately, there have been few attempts in leishmaniasis to tackle the innumerable challenges to standardize laboratory procedures from the initial stages of parasite isolation and sample propagation, even though some basic variability can never be avoided (Fig. 1). While the introduction of an activity index provided a useful tool to compare experiments between laboratories and between different experiment series (Yardley et al. 2006), implementation of stricter 'drug susceptibility procedures' will add to the quality of monitoring programmes in the field and even facilitate discovery research for novel antileishmanial drugs. Despite several calls for standardization in the past (Croft, 2001; Croft et al. 2006), endpoint criteria and assays required to obtain qualitative data have so far only been defined for natural products (Cos et al. 2006), although the same rules could obviously also be used for small molecules.

\section{Launching a harmonization initiative}

The added value of standardized Leishmania research procedures is obvious. The first required step is the creation of task force or working group to bring experts from academia, diagnostic laboratories, the clinic and public health together with those who have pioneered similar programmes in malaria and microbial infections, to establish and disseminate a critical set of procedures and analytical tools that will define acceptable levels of harmonization and uniformity between different laboratories. To start this process, a first proposal of some basic procedures concerning diagnostic sampling and follow-up standard laboratory procedures is presented in the Supplementary Material Section (Table 4). The various efforts by the WWARN platform enabled a better comprehension of the factors affecting drug efficacy which subsequently has been corrected by policy changes. For example, WWARN developed a unique on-line model (http://www.wwarn.org/toolsresources/external-quality-assurance) to facilitate individual patient data sharing and engaged over 260 partners around the world, comprised research institutions, governmental and non-governmental organizations, product development partnerships and pharmaceutical companies. These examples of impact should encourage a prompt response from the Leishmania research community and launch a similar action plan in support of the elimination of VL from India, Nepal and Bangladesh by 2017 as public health priority (WHO, 2015).

Beyond this initial action, there are still some aspects that deserve particular consideration in drug resistance research. Given the paucity of drug-resistant clinical isolates, resistance research for most drugs has mainly relied on laboratory-selected strains or on unmatched clinical isolates, hence drifting away from a comparable genetic and phenotypic background. The lack of paired pre- and post-treatment isolates obscures correct interpretation of shifting drug susceptibility. Additionally, the long adaptation process from initial isolation until evaluation in the laboratory further impairs the acquisition of representative clinical isolates, partly related to a changing virulence and genomic profile (Silva et al. 2011; Moreira et al. 2012). Although previous research already proposed preconditioning of promastigotes as a way to increase and synchronize infectivity and virulence in vitro (Inocencio da Luz et al. 2009), its systematic use may be debatable as it will affect the original strainspecific phenotypic characteristics.

As already stated, most research has focused on $\mathrm{Sb}^{\mathrm{V}}$-resistance as large numbers of Sb-resistant clinical isolates were indeed available in India, making laboratory selection of Sb-resistance uncommon. However, as there are relatively few novel antileishmanial compounds in development (www.dndi.org), greater vigilance is warranted and attention must be paid to proactively address treatment failures and relapses in an attempt to contain the risk of drug resistance. Laboratory selection of resistance strains has long been established as a valid tool to study phenotypic changes accompanied by the selection of drug resistance and to unravel underlying resistance mechanisms. The most important benefit of experimental resistance selection is the availability of matched pairs in which the acquired drug resistance undeniably results from the incurred genomic, proteomic and phenotypic variations. However, the relevance of laboratory selected resistant strains exposed to constant drug concentrations in plastic 
vessels to the 'real world' pharmacokinetic variation of drug concentration and different physiological conditions (e.g. oxygen tension) in different tissues has not been established (MacGowan et al. 2001).

Many laboratories still select for drug resistance on the promastigote vector stage by cyclic exposure to serially increasing drug concentrations (Maarouf et al. 1998; Seifert et al. 2003; Perez-Victoria et al. 2006b; Bhandari et al. 2014). Selection on axenic promastigotes has already proven to result in different resistance mechanisms than the ones observed in the clinical setting (Goyeneche-Patino et al. 2008). Although much more complex and laborious, selection of MIL- and PMM-resistance at the intracellular amastigote level has proven successful both in vitro and in vivo (Hendrickx et al. 2012, 2015c). While the lack of active amastigote replication during successive in vitro treatment cycles could suggest passive selection of less susceptible strains (Hendrickx et al. 2015d), the protocoldependent outcome for PMM strongly endorses the need to use intracellular amastigotes in drug resistance research (Hendrickx et al. 2014, 2012) or at least reach for proper validation when using promastigotes. While overlooking the potential involvement of sand fly factors in transmission and infectivity (Bates, 2007) and still requiring largescale validation with actual resistant clinical isolates, the intracellular amastigote laboratory models do offer a more representative and predictive alternative to promastigote-based models. Although MILresistance could be generated relatively easily at the promastigote level (Perez-Victoria et al. 2003a; Seifert et al. 2003), it should be mentioned that the amastigote-based protocols resulted in the selection of only one MIL-resistant isolate of L. infantum (Hendrickx et al. 2015d), which might be suggestive for strain/species-specificity.

POSSIBLE EPIDEMIOLOGIC IMPLICATIONS OF RESISTANCE

Selection of drug resistance in most organisms generally results in particular disadvantages with regard to successful survival, reproduction and/or transmission between hosts in a given environment, better known as 'fitness' (Natera et al. 2007; Borrell \& Gagneux, 2009; Orr, 2009; Ait-Oudhia et al. 2011). For Leishmania, fitness is mainly reflected by the parasite's growth potential, infectivity and its ability to be transmitted (Natera et al. 2007). The specific impact of resistance remains debatable and likely depends on the particular drug and parasite species (Kink \& Chang, 1987; Detke et al. 1988; Gazola et al. 2001; Al-Mohammed et al. 2005). Several studies indicated enhanced infectivity, metacyclogenesis and transmission in Sbresistant parasites (Vanaerschot et al. 2012a). Next to the high selection pressure associated with anthroponotic transmission, this increased parasite fitness might partly explain the widespread Sbresistance in the Indian subcontinent (Ouakad et al. 2011; Vanaerschot et al. 2011, 2010). As already mentioned, correct interpretation of such studies may be challenging since they compared sample sets of unpaired isolates with different genotypic and phenotypic background traits (Laurent et al. 2007). Since Leishmania strains are heterogeneous and show genomic plasticity, the use of wild-type (WT) parent and directly derived drugresistant lines will rule out the involvement of factors other than an altered drug susceptibility phenotype. At present, this has only been achieved with laboratory-selected resistant strains.

For PMM, increased fitness was observed in a L. donovani promastigote-selected strain (Bhandari et al. 2014) and further corroborated in an in vitro L. infantum amastigote-selected clinical isolate (Hendrickx et al. 2016). However, in the latter study no obvious fitness benefit could be detected in promastigotes, once again endorsing again the protocol-dependent and possibly species-dependent outcome of selection (Hendrickx et al. 2016). The potential fitness benefit on intracellular amastigote replication and the enhanced defence against oxidative and nitrosative stress, combined with the fairly rapid selection of PMM-resistance (Hendrickx et al. 2014, 2012), endorse that PMM should only be used in combination therapy. On the other hand for MIL, resistance selection on amastigotes revealed that resistance may arise much slower than originally anticipated (Hendrickx et al. 2014) and that contrary to previous findings (Rai et al. 2013), the spread of primary MIL-resistant parasites in the field may be hampered by fitness disadvantages (Hendrickx et al. 2016). Although the possibility of decreased fitness may seem somewhat reassuring, the increasing number of MIL-treatment failures is nevertheless alarming particularly since the underlying nature of treatment failure still remains largely unidentified. Studies suggest that treatment failures might be linked to MIL's pharmacokinetic properties, poor compliance linked to the long treatment regimen and/or a reduced drug exposure (Rijal et al. 2013; Dorlo et al. 2014). However, repetitive MIL-treatment in relapse patients would certainly expedite the emergence of primary MIL-resistance. This is particularly worrying for $L$. infantum, which is highly prevalent in HIV co-infected patients and in canL, both conditions which are repeatedly treated with MIL even though complete parasite eradication cannot be achieved, thereby creating a huge parasite reservoir subject to MIL-exposure and resistance selection (Noli \& Saridomichelakis, 2014; van Griensven et al. 2014). Of particular concern is that the few available MIL-resistant isolates (either clinical isolates or in vitro selected on the amastigote level) are indeed all L. infantum. 


\section{Concluding remarks}

Monitoring and surveillance of drug sensitivity and resistance are essential to safeguard current treatment options and to establish models for the introduction of new drugs in the future. To discriminate resistant and susceptible strains, a clear definition of drug resistance with establishment of resistance 'breakpoints' is definitely needed. Since no validated genomic resistance markers are yet available, resistance monitoring will continue to depend on standard in vitro parasite susceptibility testing that should focus on the intracellular amastigote whenever possible and better standardized in vivo assessment. The current lack of shared procedures should be addressed with priority to allow correct interpretation and comparison of drug susceptibility studies between labs. In addition, monitoring and surveillance of Leishmania resistance in the field should definitely include large sample sizes of matched pretreatment and post-treatment isolates.

Large-scale implementation of particular SOPs will be challenging and require a time and logistic organization. The present paper is a first tentative proposal to launch a harmonization initiative for particular basic and applied Leishmania research procedures. Obviously, this proposed set will have to be disseminated and discussed with the whole Leishmania research community at international conferences and within network groups (for example, WorldLeish) to acquire input and practical feedback to enable a widely feasible set of recommendations. Through collaborations with the WHO Neglected Tropical Diseases Department (WHO-NTD) and the WHO's Special Programme for Research and Training in Tropical Diseases (WHO-TDR), performance of elimination and control programmes at country level may become enhanced. A special consortium has already been founded by the Bill and Melinda Gates Foundation to ensure suitable surveillance measures to sustain VL elimination In the Indian subcontinent. Joining all these various efforts should eventually pay off and lead to more reliable and comparable laboratory results that will inform the leishmaniasis community worldwide on the efficacy of the current antileishmanial drugs and the emergence and spread of drug resistance.

\section{SUPPLEMENTARY MATERIAL}

The supplementary material for this article can be found at https://doi.org/10.1017/S0031182016002031

\section{ACKNOWLEDGEMENTS}

The authors want to thank all of the collaborators of the European Commission's Seventh Framework Programme (Kaladrug-R project, Grant 222895).
LMPH is partner of the Antwerp Drug Discovery Network (ADDN).

FINANCIAL SUPPORT

This research received no specific grant from any funding agency, commercial or not-for-profit sectors.

\section{REFERENCES}

Ait-Oudhia, K., Gazanion, E., Vergnes, B., Oury, B. and Sereno, D. (2011). Leishmania antimony resistance: what we know what we can learn from the field. Parasitology Research 109, 1225-1232.

Al-Mohammed, H. I., Chance, M. L. and Bates, P.A. (2005) Production and characterization of stable amphotericin-resistant amastigotes and promastigotes of Leishmania mexicana. Antimicrobial Agents and Chemotherapy 49, 3274-3280.

Alvar, J., Vélez, I. D., Bern, C., Herrero, M., Desjeux, P., Cano, J., Jannin, J., den Boer, M., WHO Leishmaniasis Control Team (2012). Leishmaniasis worldwide and global estimates of its incidence. PLoS ONE 7, e35671.

Ashutosh, Sundar, S. and Goyal, N. (2007). Molecular mechanisms of antimony resistance in Leishmania. Fournal of Medical Microbiology 56, 143-153.

Ashutosh, Garg, M., Sundar, S., Duncan, R., Nakhasi, H. L. and Goyal, N. (2012). Downregulation of mitogen-activated protein kinase 1 of Leishmania donovani field isolates is associated with antimony resistance. Antimicrobial Agents and Chemotherapy 56, 518-525.

Balasegaram, M., Ritmeijer, K., Lima, M. A., Burza, S., Ortiz Genovese, G., Milani, B., Gaspani, S., Potet, J., Chappuis, F. (2012). Liposomal amphotericin B as a treatment for human leishmaniasis. Expert Opinion on Emerging Drugs 17, 493-510.

Bates, P. A. (2007). Transmission of Leishmania metacyclic promastigotes by phlebotomine sand flies. International fournal for Parasitology 37, 1097-1106.

Berg, M., Vanaerschot, M., Jankevics, A., Cuypers, B., Maes, I., Mukherjee, S., Khanal, B., Rijal, S., Roy, S., Opperdoes, F., Breitling, R. and Dujardin, J.C. (2013). Metabolic adaptations of Leishmania donovani in relation to differentiation, drug resistance, and drug pressure. Molecular Microbiology 90, 428-442.

Bhandari, V., Kulshrestha, A., Deep, D. K., Stark, O., Prajapati, V. K., Ramesh, V., Sundar, S., Schonian, G., Dujardin, J. C. and Salotra, P. (2012). Drug susceptibility in Leishmania isolates following miltefosine treatment in cases of visceral leishmaniasis and post kala-azar dermal leishmaniasis. PLoS Neglected Tropical Diseases 6, e1657.

Bhandari, V., Sundar, S., Dujardin, J. C. and Salotra, P. (2014). Elucidation of cellular mechanisms involved in experimental paromomycin resistance in Leishmania donovani. Antimicrobial Agents and Chemotherapy 58, 2580-2585.

Bhunia, G. S., Kesari, S., Chatterjee, N., Kumar, V., Das, P. (2013) Spatial and temporal variation and hotspot detection of kala-azar disease in Vaishali district (Bihar), India. BMC Infectious Diseases BMC Series 13, 64 Blackwell, J. M. (1996). Genetic susceptibility to leishmanial infections: studies in mice and man. Parasitology, 112 (Suppl), S67-S74.

Boelaert, M., Verdonck, K., Menten, J., Sunyoto, T., van, G. J., Chappuis, F. and Rijal, S. (2014). Rapid tests for the diagnosis of visceral leishmaniasis in patients with suspected disease. Cochrane Database of Systematic Reviews, 6, CD009135.

Boothe, D. M. (2010). Interpreting culture and susceptibility data in critical care: perks and pitfalls. Journal of Veterinary Emergency and Critical Care (San.Antonio.), 20, 110-131.

Borrell, S. and Gagneux, S. (2009). Infectiousness, reproductive fitness and evolution of drug-resistant Mycobacterium tuberculosis. International Fournal of Tuberculosis and Lung Disease 13, 1456-1466.

Brotherton, M. C., Bourassa, S., Leprohon, P., Legare, D., Poirier, G. G., Droit, A. and Ouellette, M. (2013). Proteomic and genomic analyses of antimony resistant Leishmania infantum mutant. PLoS ONE 8, e81899. Carnielli, J. B., de Andrade, H. M., Pires, S. F., Chapeaurouge, A. D., Perales, J., Monti-Rocha, R., Carvalho, S. F., Ribeiro, L.P., Dietze, R., Figueiredo, S. G. and Lemos, E. M. (2014). Proteomic analysis of the soluble proteomes of miltefosine-sensitive and -resistant Leishmania infantum chagasi isolates obtained from Brazilian patients with different treatment outcomes. Fournal of Proteomics 108, 198-208. Castellucci, L.C., Almeida, L.F., Jamieson, S.E., Fakiola, M., Carvalho, E. M. and Blackwell, J.M. (2014). Host genetic factors in 
American cutaneous leishmaniasis: a critical appraisal of studies conducted in an endemic area of Brazil. Memórias do Instituto Oswaldo Cruz 109, 279-288. Chakravarty, J. and Sundar, S. (2010). Drug resistance in leishmaniasis. Fournal of Global Infectious Diseases 2, 167-176.

Chrusciak-Talhari, A., Dietze, R., Chrusciak Talhari, C., da Silva, R. M., Gadelha Yamashita, E.P., de Oliveira Penna, G., Lima Machado, P. R. and Talhari, S. (2011). Randomized controlled clinical trial to access efficacy and safety of miltefosine in the treatment of cutaneous leishmaniasis Caused by Leishmania (Viannia) guyanensis in Manaus, Brazil. The American Fournal of Tropical Medicine and Hygiene 84, 255-260.

Cos, P., Vlietinck, A. J., Berghe, D. V. and Maes, L. (2006). Anti-infective potential of natural products: how to develop a stronger in vitro 'proofof-concept'. Fournal of Ethnopharmacology 106, 290-302.

Croft, S. L. (2001). Monitoring drug resistance in leishmaniasis. Tropical Medicine Eீ International Health 6, 899-905.

Croft, S. L., Sundar, S. and Fairlamb, A. H. (2006). Drug resistance in leishmaniasis. Clinical Microbiology Reviews 19, 111-126.

Davidson, R. N., den Boer, M., Ritmeijer, K. (2009). Paromomycin. Transactions of the Royal Society of Tropical Medicine and Hygiene 103, 653-660.

De Jesus, J. B., Mesquita-Rodrigues, C. and Cuervo, P. (2014). Proteomics advances in the study of Leishmania parasites and leishmaniasis. Subcellular Biochemistry 74, 323-349.

Detke, S., Chaudhuri, G., Kink, J. A. and Chang, K. P. (1988). DNA amplification in tunicamycin-resistant Leishmania mexicana. Multicopies of a single 63-kilobase supercoiled molecule and their expression. Fournal of Biological Chemistry 263, 3418-3424.

Dorlo, T. P., Eggelte, T. A., de Vries, P. J. and Beijnen, J. H. (2012a). Characterization and identification of suspected counterfeit miltefosine capsules. Analyst 137, 1265-1274

Dorlo, T.P., Eggelte, T.A., Schoone, G. J., de Vries, P. J. and Beijnen, J. H. (2012b). A poor-quality generic drug for the treatment of visceral leishmaniasis: a case report and appeal. PLoS Neglected Tropical Diseases 6, e1544.

Dorlo, T.P., Rijal, S., Ostyn, B., de Vries, P. J., Singh, R., Bhattarai, N., Uranw, S., Dujardin, J. C., Boelaert, M., Beijnen, J. H. and Huitema, A. D. (2014). Failure of miltefosine in visceral Leishmaniasis is associated with low drug exposure. The fournal of Infectious Diseases 210, 146-153.

Downing, T., Imamura, H., Decuypere, S., Clark, T. G., Coombs, G. H., Cotton, J. A., Hilley, J. D., De, D. S., Maes, I., Mottram, J. C., Quail, M. A., Rijal, S., Sanders, M., Schonian, G., Stark, O., Sundar, S., Vanaerschot, M., Hertz-Fowler, C., Dujardin, J. C. and Berriman, M. (2011). Whole genome sequencing of multiple Leishmania donovani clinical isolates provides insights into population structure and mechanisms of drug resistance. Genome Research 21, 2143-2156.

Duru, V., Khim, N., Leang, R., Kim, S., Domergue, A., Kloeung, N., Ke, S., Chy, S., Eam, R., Khean, C., Loch, K., Ken, M., Lek, D., Beghain, J., Ariey, F., Guerin, P. J., Huy, R., MercereauPuijalon, O., Witkowski, B. and Menard, D. (2015). Plasmodium falciparum dihydroartemisinin-piperaquine failures in Cambodia are associated with mutant K13 parasites presenting high survival rates in novel piperaquine in vitro assays: retrospective and prospective investigations. $B M C$ Medicine 13, 305

El, F. K., Drummelsmith, J., Roy, G., Jardim, A. and Ouellette, M. (2009). Down regulation of KMP-11 in Leishmania infantum axenic antimony resistant amastigotes as revealed by a proteomic screen. Experimental Parasitology 123, 51-57.

Fernandez, O., Diaz-Toro, Y., Valderrama, L., Ovalle, C., Valderrama, M., Castillo, H., Perez, M. and Saravia, N. G. (2012). Novel approach to in vitro drug susceptibility assessment of clinical strains of Leishmania spp. Fournal of Clinical Microbiology 50, 2207-2211. Fumarola, L., Spinelli, R. and Brandonisio, O. (2004). In vitro assays for evaluation of drug activity against Leishmania spp. Research in Microbiology 155, 224-230.

Garcia-Hernandez, R., Manzano, J. I., Castanys, S. and Gamarro, F. (2012). Leishmania donovani develops resistance to drug combinations. PLoS Neglected Tropical Diseases 6, e1974.

Gazola, K. C., Ferreira, A. V., Anacleto, C., Michalick, M.S., Andrade, A. F. and Moreira, E. S. (2001). Cell surface carbohydrates and in vivo infectivity of glucantime-sensitive and resistant Leishmania (Viannia) guyjanensis cell lines. Parasitology Research 87, 935-940.

Geli, P., Laxminarayan, R., Dunne, M. and Smith, D. L. (2012). "One-size-fits-all"? Optimizing treatment duration for bacterial infections. PLoS ONE 7, e29838.

Gouzelou, E., Haralambous, C., Antoniou, M., Christodoulou, V., Martinkovic, F., Zivicnjak, T., Smirlis, D., Pratlong, F., Dedet, J.
P., Ozbel, Y., Toz, S. O., Presber, W., Schonian, G. and Soteriadou, K. (2013). Genetic diversity and structure in Leishmania infantum populations from southeastern Europe revealed by microsatellite analysis. Parasites \& Vectors 6, 342

Goyeneche-Patino, D. A., Valderrama, L., Walker, J. and Saravia, N. G. (2008). Antimony resistance and trypanothione in experimentally selected and clinical strains of Leishmania panamensis. Antimicrobial Agents and Chemotherapy 52, 4503-4506.

Gupta, N., Goyal, N. and Rastogi, A. K. (2001). In vitro cultivation and characterization of axenic amastigotes of Leishmania. Trends in Parasitology 17, 150-153

Gurunath, U., Joshi, R., Agrawal, A. and Shah, V. (2014). An overview of visceral leishmaniasis elimination program in India: a picture imperfect. Expert Review of Anti-infective Therapy 12, 929-935.

Hailu, A., Musa, A., Wasunna, M., Balasegaram, M., Yifru, S., Mengistu, G., Hurissa, Z., Hailu, W., Weldegebreal, T., Tesfaye, S., Makonnen, E., Khalil, E., Ahmed, O., Fadlalla, A., ElHassan, A., Raheem, M., Mueller, M., Koummuki, Y., Rashid, J., Mbui, J., Mucee, G., Njoroge, S., Manduku, V., Musibi, A., Mutuma, G., Kirui, F., Lodenyo, H., Mutea, D., Kirigi, G., Edwards, T., Smith, P., Muthami, L., Royce, C., Ellis, S., Alobo, M., Omollo, R., Kesusu, J., Owiti, R. and Kinuthia, J. (2010). Geographical variation in the response of visceral leishmaniasis to paromomycin in East Africa: a multicentre, open-label, randomized trial. PLoS Neglected Tropical Diseases 4, e709.

Haldar, A. K., Sen, P. and Roy, S. (2011). Use of antimony in the treatment of leishmaniasis: current status and future directions. Molecular Biology International 2011, 571242.

Hastings, I. M., Watkins, W. M. and White, N. J. (2002). The evolution of drug-resistant malaria: the role of drug elimination half-life. Philosophical Transactions of the Royal Society of London. Series B, Biological Sciences 357(1420), 505-519.

Hendrickx, S., Inocencio da Luz, R. A., Bhandari, V., Kuypers, K., Shaw, C. D., Lonchamp, J., Salotra, P., Carter, K., Sundar, S., Rijal, S., Dujardin, J. C., Cos, P. and Maes, L. (2012). Experimental induction of paromomycin resistance in antimony-resistant strains of $L$. donovani: outcome dependent on in vitro selection protocol. PLoS Neglected Tropical Diseases 6, e1664.

Hendrickx, S., Boulet, G., Mondelaers, A., Dujardin, J. C., Rijal, S., Lachaud, L., Cos, P., Delputte, P. and Maes, L. (2014). Experimental selection of paromomycin and miltefosine resistance in intracellular amastigotes of Leishmania donovani and L. infantum. Parasitology Research 113, 1875-1881.

Hendrickx, S., Eberhardt, E., Mondelaers, A., Rijal, S., Bhattarai, N. R., Dujardin, J. C., Delputte, P., Cos, P. and Maes, L. (2015a). Lack of correlation between the promastigote back-transformation assay and miltefosine treatment outcome. Fournal of Antimicrobial Chemotherapy 70, 3023-3026.

Hendrickx, S., Leemans, A., Mondelaers, A., Rijal, S., Khanal, B., Dujardin, J. C., Delputte, P., Cos, P., Maes, L. (2015b). Comparative fitness of a parent Leishmania donovani clinical isolate and its experimentally derived paromomycin-resistant strain. PLoS ONE 10, e0140139.

Hendrickx, S., Mondelaers, A., Eberhardt, E., Delputte, P., Cos, P. and Maes, L. (2015c). In vivo selection of paromomycin and miltefosine resistance in Leishmania donovani and L. infantum in a Syrian Hamster Model. Antimicrobial Agents and Chemotherapy 59, 4714-4718.

Hendrickx, S., Mondelaers, A., Eberhardt, E., Lachaud, L. Delputte, P., Cos, P. and Maes, L. (2015d). Intracellular amastigote replication may not be required for successful in vitro selection of miltefosine resistance in Leishmania infantum. Parasitology Research 114, 2561-2565. Hendrickx, S., Beyers, J., Mondelaers, A., Eberhardt, E., Lachaud, L., Delputte, P., Cos, P. and Maes, L. (2016). Evidence of a drug-specific impact of experimentally selected paromomycin and miltefosine resistance on parasite fitness in Leishmania infantum. Fournal of Antimicrobial Chemotherapy 71, 1914-1921.

Houweling, T.A., Karim-Kos, H.E., Kulik, M.C., Stolk, W.A., Haagsma, J. A., Lenk, E. J., Richardus, J. H., de Vlas, S. J. (2016). Socioeconomic inequalities in neglected tropical diseases: a systematic review. PLoS Neglected Tropical Diseases 10, e0004546.

Imamura, H., Downing, T., Van den Broeck, F., Sanders, M. J. Rijal, S., Sundar, S., Mannaert, A., Vanaerschot, M., Berg, M., De Muylder, G., Dumetz, F., Cuypers, B., Maes, I., Domagalska, M., Decuypere, S., Rai, K., Uranw, S., Bhattarai, N. R., Khanal, B., Prajapati, V. K., Sharma, S., Stark, O., Schönian, G., De Koning, H.P., Settimo, L., Vanhollebeke, B., Roy, S., Ostyn, B., Boelaert, M., Maes, L., Berriman, M., Dujardin, J. C., Cotton, J. A. (2016). Evolutionary genomics of epidemic visceral leishmaniasis in the Indian subcontinent. Elife 5, e12613. doi: 10.7554/eLife.12613. 
Inocencio da Luz, R. A., Vermeersch, M., Dujardin, J. C., Cos, P. and Maes, L. (2009). In vitro sensitivity testing of Leishmania clinical field isolates: preconditioning of promastigotes enhances infectivity for macrophage host cells. Antimicrobial Agents and Chemotherapy 53, 5197-5203. Jarvis, J. N. and Lockwood, D. N. (2013). Clinical aspects of visceral leishmaniasis in HIV infection. Current Opinion in Infectious Diseases, 26, 1-9. Jha, R. K., Sah, A. K., Shah, D. K., Sah, P. (2013). The treatment of visceral leishmaniasis: safety and efficacy. Fournal of the Nepalese Medical Association 52, 645-651.

Kazemi-Rad, E., Mohebali, M., Khadem-Erfan, M. B., Saffari, M. Raoofian, R., Hajjaran, H., Hadighi, R., Khamesipour, A., Rezaie, S., Abedkhojasteh, H. and Heidari, M. (2013). Identification of antimony resistance markers in Leishmania tropica field isolates through a cDNA-AFLP approach. Experimental Parasitology 135, 344-349.

Kink, J. A. and Chang, K. P. (1987). Biological and biochemical characterization of tunicamycin-resistant Leishmania mexicana: mechanism of drug resistance and virulence. Infection and Immunity 55, 1692-1700. Kulshrestha, A., Bhandari, V., Mukhopadhyay, R., Ramesh, V., Sundar, S., Maes, L., Dujardin, J. C., Roy, S. and Salotra, P. (2013) Validation of a simple resazurin-based promastigote assay for the routine monitoring of miltefosine susceptibility in clinical isolates of Leishmania donovani. Parasitology Research 112, 825-828.

Kumar, D., Singh, R., Bhandari, V., Kulshrestha, A., Negi, N. S. and Salotra, P. (2012). Biomarkers of antimony resistance: need for expression analysis of multiple genes to distinguish resistance phenotype in clinical isolates of Leishmania donovani. Parasitology Research 111, 223-230.

Laurent, T., Rijal, S., Yardley, V., Croft, S., De, D. S., Decuypere, S., Khanal, B., Singh, R., Schonian, G., Kuhls, K., Chappuis, F. and Dujardin, J. C. (2007). Epidemiological dynamics of antimonial resistance in Leishmania donovani: genotyping reveals a polyclonal population structure among naturally-resistant clinical isolates from Nepal. Infection, Genetics and Evolution 7, 206-212.

Leprohon, P., Legare, D., Raymond, F., Madore, E., Hardiman, G., Corbeil, J. and Ouellette, M. (2009). Gene expression modulation is associated with gene amplification, supernumerary chromosomes and chromosome loss in antimony-resistant Leishmania infantum. Nucleic Acids Research 37, 1387-1399.

Lourens, C., Watkins, W. M., Barnes, K. I., Sibley, C. H., Guerin, P. J., White, N. J., Lindegardh, N. (2010). Implementation of a reference standard and proficiency testing programme by the World Wide Antimalarial Resistance Network (WWARN). Malar Fournal 9, 375. Lourens, C., Lindegardh, N., Barnes, K. I., Guerin, P. J., Sibley, C. H., White, N. J., Tarning, J. (2014). Benefits of a pharmacology antimalarial reference standard and proficiency testing program provided by the Worldwide Antimalarial Resistance Network (WWARN). Antimicrob Agents Chemother 58, 3889-3894.

Maarouf, M., Adeline, M. T., Solignac, M., Vautrin, D. and RobertGero, M. (1998). Development and characterization of paromomycinresistant Leishmania donovani promastigotes. Parasite 5, 167-173.

MacGowan, A., Rogers, C., Bowker, K. (2001) In Vitro models, In Vivo models, and pharmacokinetics: what can we learn from in vitro models? Clinical Infectectious Diseases 33 (Suppl. 3), S214-S220.

Machado, P. R., Ampuero, J., Guimaraes, L.H., Villasboas, L., Rocha, A. T., Schriefer, A., Sousa, R. S., Talhari, A., Penna, G. and Carvalho, E. M. (2010). Miltefosine in the treatment of cutaneous leishmaniasis caused by Leishmania braziliensis in Brazil: a randomized and controlled trial. PLoS Neglected Tropical Diseases 4, e912.

Maes, L., Cos, P. and Croft, S. (2013). The relevance of susceptibility tests, breakpoints and markers. In Drug Resistance in Leishmania Parasites (eds. Ponte-Sucre, A., Diaz, E. and Padrón-Nieves, M.), pp. 407-429. Springer, Vienna.

Mandal, S., Maharjan, M., Singh, S., Chatterjee, M. and Madhubala, R. (2010). Assessing aquaglyceroporin gene status and expression profile in antimony-susceptible and -resistant clinical isolates of Leishmania donovani from India. Fournal of Antimicrobial Chemotherapy 65, 496-507.

Mittal, M. K., Rai, S., Ashutosh, R., Gupta, S., Sundar, S. and Goyal, N. (2007). Characterization of natural antimony resistance in Leishmania donovani isolates. The American Fournal of Tropical Medicine and Hygiene 76, 681-688.

Mondal, S., Bhattacharya, P. and Ali, N. (2010). Current diagnosis and treatment of visceral leishmaniasis. Expert Review of Anti-infective Therapy 8, 919-944.

Mondelaers, A., Sanchez-Cañete, M.P., Hendrickx, S., Eberhardt, E., Garcia-Hernandez, R., Lachaud, L., Cotton, J., Sanders, M., Cuypers, B., Imamura, H., Dujardin, J. C., Delputte, P., Cos, P., Caljon, G., Gamarro, F., Castanys, S. and Maes, L. (2016). Genomic and molecular characterization of miltefosine resistance in Leishmania infantum strains with either natural or acquired resistance through experimental selection of intracellular amastigotes. PLoS ONE 11, e0154101.

Moreira, D., Santarem, N., Loureiro, I., Tavares, J., Silva, A. M. Amorim, A. M., Ouaissi, A., Cordeiro-da-Silva, A. and Silvestre, R. (2012). Impact of continuous axenic cultivation in Leishmania infantum virulence. PLoS Neglected Tropical Diseases 6, e1469.

Mukherjee, A., Padmanabhan, P. K., Singh, S., Roy, G., Girard, I., Chatterjee, M., Ouellette, M. and Madhubala, R. (2007). Role of ABC transporter MRPA, gamma-glutamylcysteine synthetase and ornithine decarboxylase in natural antimony-resistant isolates of Leishmania donovani. Fournal of Antimicrobial Chemotherapy 59, 204-211.

Mukherjee, B., Mukhopadhyay, R., Bannerjee, B., Chowdhury, S., Mukherjee, S., Naskar, K., Allam, U.S., Chakravortty, D., Sundar, S., Dujardin, J. C. and Roy, S. (2013). Antimony-resistant but not antimony-sensitive Leishmania donovani up-regulates host IL-10 to overexpress multidrug-resistant protein 1. Proceedings of the National Academy of Sciences of the United States of America 110, E575-E582.

Muniaraj, M. (2014). The lost hope of elimination of kala-azar (visceral leishmaniasis) by 2010 and cyclic occurrence of its outbreak in India, blame falls on vector control practices or co-infection with human immunodeficiency virus or therapeutic modalities? Tropical Parasitology 4, 10-19.

Natera, S., Machuca, C., Padron-Nieves, M., Romero, A., Diaz, E. and Ponte-Sucre, A. (2007). Leishmania spp.: proficiency of drug-resistant parasites. International Fournal of Antimicrobial Agents 29, 637-642. Noli, C. and Saridomichelakis, M. N. (2014). An update on the diagnosis and treatment of canine leishmaniosis caused by Leishmania infantum (syn. L. chagasi). The Veterinary fournal 202, 425-435.

Orr, H. A. (2009). Fitness and its role in evolutionary genetics. Nature Reviews Genetics 10, 531-539.

Ostyn, B., Hasker, E., Dorlo, T. P., Rijal, S., Sundar, S., Dujardin, J. C. and Boelaert, M. (2014). Failure of miltefosine treatment for visceral leishmaniasis in children and men in South-East Asia. PLoS ONE 9 , e100220.

Ouakad, M., Vanaerschot, M., Rijal, S., Sundar, S., Speybroeck, N., Kestens, L., Boel, L., De, D.S., Maes, I., Decuypere, S. and Dujardin, J. C. (2011). Increased metacyclogenesis of antimony-resistant Leishmania donovani clinical lines. Parasitology 138, 1392-1399.

Perez-Victoria, F. J., Castanys, S. and Gamarro, F. (2003a) Leishmania donovani resistance to miltefosine involves a defective inward translocation of the drug. Antimicrobial Agents and Chemotherapy 47 2397-2403.

Perez-Victoria, F. J., Gamarro, F., Ouellette, M. and Castanys, S. (2003b). Functional cloning of the miltefosine transporter. A novel Ptype phospholipid translocase from Leishmania involved in drug resistance. The Fournal of Biological Chemistry 278, 49965-49971.

Perez-Victoria, F.J., Sanchez-Canete, M.P., Castanys, S. and Gamarro, F. (2006a). Phospholipid translocation and miltefosine potency require both $L$. donovani miltefosine transporter and the new protein LdRos3 in Leishmania parasites. The fournal of Biological Chemistry 281, 23766-23775.

Perez-Victoria, F. J., Sanchez-Canete, M. P., Seifert, K., Croft, S. L., Sundar, S., Castanys, S. and Gamarro, F. (2006 b). Mechanisms of experimental resistance of Leishmania to miltefosine: implications for clinical use. Drug Resistance Update 9, 26-39.

Perry, M. R., Wyllie, S., Raab, A., Feldmann, J. and Fairlamb, A. H. (2013). Chronic exposure to arsenic in drinking water can lead to resistance to antimonial drugs in a mouse model of visceral leishmaniasis. Proceedings of the National Academy of Sciences 110, 19932-19937.

Purkait, B., Kumar, A., Nandi, N., Sardar, A. H., Das, S., Kumar, S., Pandey, K., Ravidas, V., Kumar, M., De, T., Singh, D., Das, P. (2012). Mechanism of amphotericin B resistance in clinical isolates of Leishmania donovani. Antimicrobial Agents and Chemotherapy 56, 1031-1041.

Rai, K., Cuypers, B., Bhattarai, N. R., Uranw, S., Berg, M., Ostyn, B., Dujardin, J. C., Rijal, S. and Vanaerschot, M. (2013). Relapse after treatment with miltefosine for visceral leishmaniasis is associated with increased infectivity of the infecting Leishmania donovani strain. MBio. 4, e00611-e00613.

Ready, P. D. (2014). Epidemiology of visceral leishmaniasis. Fournal of Clinical Epidemiology, 6, 147-154.

Rex, J.H., Pfaller, M. A., Galgiani, J. N., Bartlett, M.S., EspinelIngroff, A., Ghannoum, M. A., Lancaster, M., Odds, F. C., Rinaldi, M. G., Walsh, T. J. and Barry, A. L. (1997). Development of interpretive breakpoints for antifungal susceptibility testing: conceptual framework and analysis of in vitro-in vivo correlation data for fluconazole, itraconazole, and candida infections. Subcommittee on Antifungal Susceptibility Testing of the National Committee for Clinical Laboratory Standards. Clinical Infectious Diseases 24, 235-247. 
Rijal, S., Ostyn, B., Uranw, S., Rai, K., Bhattarai, N. R., Dorlo, T.P., Beijnen, J.H., Vanaerschot, M., Decuypere, S., Dhakal, S. S., Das, M. L., Karki, P., Singh, R., Boelaert, M. and Dujardin, J. C. (2013). Increasing failure of miltefosine in the treatment of kala-azar in Nepal and the potential role of parasite drug resistance, reinfection, or noncompliance. Clinical Infectious Diseases 56, 1530-1538.

Scheltema, R.A., Decuypere, S., t'Kindt, R., Dujardin, J.C., Coombs, G. H. and Breitling, R. (2010). The potential of metabolomics for Leishmania research in the post-genomics era. Parasitology 137, 1291-1302. Seifert, K., Matu, S., Javier Perez-Victoria, F., Castanys, S., Gamarro, F. and Croft, S. L. (2003). Characterisation of Leishmania donovani promastigotes resistant to hexadecylphosphocholine (miltefosine). International Fournal of Antimicrobial Agents 22, 380-387.

Seifert, K., Perez-Victoria, F. J., Stettler, M., Sanchez-Canete, M. P., Castanys, S., Gamarro, F. and Croft, S. L. (2007). Inactivation of the miltefosine transporter, LdMT, causes miltefosine resistance that is conferred to the amastigote stage of Leishmania donovani and persists in vivo. International fournal of Antimicrobial Agents 30, 229-235.

Seifert, K., Escobar, P. and Croft, S. L. (2010). In vitro activity of antileishmanial drugs against Leishmania donovani is host cell dependent. Fournal of Antimicrobial Chemotherapy 65, 508-511.

Sibley, C. H. and Price, R. N. (2012). Monitoring antimalarial drug resistance: applying lessons learned from the past in a fast-moving present. International Fournal for Parasitology: Drugs and Drug Resistance 2, 126-133

Silva, A. M., Cordeiro-da-Silva, A. and Coombs, G. H. (2011). Metabolic variation during development in culture of Leishmania donovani promastigotes. PLoS Neglected Tropical Diseases 5, e1451.

Singh, N. (2006). Drug resistance mechanisms in clinical isolates of Leishmania donovani. Indian Fournal of Medical Research 123, 411-422.

Singh, R., Kumar, D., Ramesh, V., Negi, N. S., Singh, S. and Salotra, P. (2006). Visceral leishmaniasis, or kala azar (KA): high incidence of refractoriness to antimony is contributed by anthroponotic transmission via post-KA dermal leishmaniasis. Fournal of Infectious Diseases 194, 302-306.

Singh, R., Kumar, D., Duncan, R. C., Nakhasi, H. L. and Salotra, P. (2010). Overexpression of histone H2A modulates drug susceptibility in Leishmania parasites. International Fournal of Antimicrobial Agents 36, $50-57$

Sinha, R., Datta, U. and Sehgal, S. (1993). Importance of bone marrow culture for diagnosis of Kala azar. Scandinavian Fournal of Infectious Diseases 25, 787-789.

Soto, J., Arana, B. A., Toledo, J., Rizzo, N., Vega, J. C., Diaz, A., Luz, M., Gutierrez, P., Arboleda, M., Berman, J. D., Junge, K., Engel, J. and Sindermann, H. (2004). Miltefosine for new world cutaneous leishmaniasis. Clinical Infectious Diseases 38, 1266-1272.

Soto, J., Rea, J., Balderrama, M., Toledo, J., Soto, P., Valda, L. and Berman, J.D. (2008). Efficacy of miltefosine for Bolivian cutaneous leishmaniasis. The American Fournal of Tropical Medicine and Hygiene 78, 210-211.

Sundar, S., Sinha, P. K., Rai, M., Verma, D. K., Nawin, K., Alam, S., Chakravarty, J., Vaillant, M., Verma, N., Pandey, K., Kumari, P., Lal, C. S., Arora, R., Sharma, B., Ellis, S., Strub-Wourgaft, N., Balasegaram, M., Olliaro, P., Das, P., Modabber, F. (2011). Comparison of short-course multidrug treatment with standard therapy for visceral leishmaniasis in India: an open-label, non-inferiority, randomised controlled trial. Lancet 377, 477-486.

Sundar, S., Singh, A. and Singh, O. P. (2014). Strategies to overcome antileishmanial drugs unresponsiveness. Fournal of Tropical Medicine 2014, 646932.

Thakur, C.P., Kanyok, T.P., Pandey, A. K., Sinha, G.P., Zaniewski, A. E., Houlihan, H. H., Olliaro, P. (2000). A prospective randomized, comparative, open-label trial of the safety and efficacy of paromomycin (aminosidine) plus sodium stibogluconate versus sodium stibogluconate alone for the treatment of visceral leishmaniasis. Transactions of the Royal Society of Tropical Medicine and Hygiene 94, 429-431.
t'Kindt, R., Scheltema, R. A., Jankevics, A., Brunker, K., Rijal, S., Dujardin, J.C., Breitling, R., Watson, D. G., Coombs, G. H. and Decuypere, S. (2010). Metabolomics to unveil and understand phenotypic diversity between pathogen populations. PLoS Neglected Tropical Diseases 4, e904.

Ubeda, J. M., Legare, D., Raymond, F., Ouameur, A. A., Boisvert, S., Rigault, P., Corbeil, J., Tremblay, M.J., Olivier, M., Papadopoulou, B. and Ouellette, M. (2008). Modulation of gene expression in drug resistant Leishmania is associated with gene amplification, gene deletion and chromosome aneuploidy. Genome Biology 9, R115.

van Griensven, J., Carrillo, E., Lopez-Velez, R., Lynen, L. and Moreno, J. (2014). Leishmaniasis in immunosuppressed individuals. Clinical Microbiology and Infection 20, 286-299.

Vanaerschot, M., Maes, I., Ouakad, M., Adaui, V., Maes, L., De, D. S., Rijal, S., Chappuis, F., Dujardin, J. C. and Decuypere, S. (2010). Linking in vitro and in vivo survival of clinical Leishmania donovani strains. PLoS ONE 5, e12211.

Vanaerschot, M., De, D.S., Rijal, S., Maes, L., Dujardin, J. C. and Decuypere, S. (2011). Antimonial resistance in Leishmania donovani is associated with increased in vivo parasite burden. PLoS ONE 6, e23120. Vanaerschot, M., Decuypere, S., Berg, M., Roy, S. and Dujardin, J. C. (2012a). Drug-resistant microorganisms with a higher fitness - can medicines boost pathogens? Critical Reviews in Microbiology 39, 384-394.

Vanaerschot, M., Decuypere, S., Downing, T., Imamura, H., Stark, O., De, D. S., Roy, S., Ostyn, B., Maes, L., Khanal, B., Boelaert, M., Schonian, G., Berriman, M., Chappuis, F., Dujardin, J. C., Sundar, S. and Rijal, S. (2012b). Genetic markers for SSG resistance in Leishmania donovani and SSG treatment failure in visceral leishmaniasis patients of the Indian subcontinent. The Fournal of Infectious Diseases 206, 752-755.

Vanaerschot, M., Dumetz, F., Roy, S., Ponte-Sucre, A., Arevalo, J. and Dujardin, J. C. (2014). Treatment failure in leishmaniasis: drugresistance or another (epi-) phenotype? Expert Review of Anti-infective Therapy 12, 937-946.

Vergnes, B., Gourbal, B., Girard, I., Sundar, S., Drummelsmith, J. and Ouellette, M. (2007). A proteomics screen implicates HSP83 and a small kinetoplastid calpain-related protein in drug resistance in Leishmania donovani clinical field isolates by modulating drug-induced programmed cell death. Molecular \& Cellular Proteomics 6, 88-101. Vermeersch, M., da Luz, R. I., Tote, K., Timmermans, J. P., Cos, P. and Maes, L. (2009). In vitro susceptibilities of Leishmania donovani promastigote and amastigote stages to antileishmanial reference drugs: practical relevance of stage-specific differences. Antimicrobial Agents and Chemotherapy 53, 3855-3859.

Woodrow, C. J., Dahlström, S., Cooksey, R., Flegg, J. A., Le Nagard, H., Mentré, F., Murillo, C., Ménard, D., Noste, F. Sriprawat, K., Musset, L., Quashie, N. B., Lim, P., Fairhurst, R. M., Nsobya, S. L., Sinou, V., Noedl, H., Pradines, B., Johnson, J. D., Guerin, P. J., Sibley, C. H., Le Bras, J. (2013). High-throughput analysis of antimalarial susceptibility data by the WorldWide Antimalarial Resistance Network (WWARN) in vitro analysis and reporting tool. Antimicrobial Agents and Chemotherapy 57, 3121-3130.

World Health Organization (2010). Control of the Leishmaniasis: Report of a Meeting of the WHO Expert Committee on the Control of Leishmaniasis, WHO technical report series No. 949. World Health Organization, Geneva, Switzerland

World Health Organization (2015) Kala-Azar Elimination Programme: Report of a WHO Consultation of Partners, Geneva, Switzerland, 10-11 February 2015.

Yardley, V., Ortuno, N., Llanos-Cuentas, A., Chappuis, F., Doncker, S.D., Ramirez, L., Croft, S., Arevalo, J., Adaui, V., Bermudez, H., Decuypere, S. and Dujardin, J. C. (2006). American tegumentary leishmaniasis: is antimonial treatment outcome related to parasite drug susceptibility? Fournal of Infectious Diseases 194, 1168-1175. 